\title{
Neuro-Oncology and Radiogenomics: Time to Integrate?
}

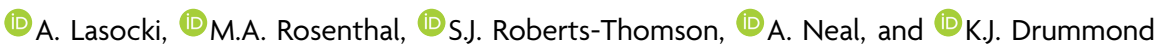

\section{ABSTRACT}

SUMMARY: Radiogenomics aims to predict genetic markers based on imaging features. The critical importance of molecular markers in the diagnosis and management of intracranial gliomas has led to a rapid growth in radiogenomics research, with progressively increasing complexity. Despite the advances in the techniques being examined, there has been little translation into the clinical domain. This has resulted in a growing disconnect between cutting-edge research and assimilation into clinical practice, though the fundamental goal is for these techniques to improve patient care. The goal of this review, therefore, is to discuss possible clinical scenarios in which the addition of radiogenomics may aid patient management. This includes facilitating patient counseling, determining optimal patient management when complete molecular characterization is not possible, reclassifying tumors, and overcoming some of the limitations of histologic assessment. The review also discusses considerations for selecting relevant radiogenomic features based on the clinical setting.

ABBREVIATIONS: IDH $=$ isocitrate dehydrogenase; IDH ${ }^{\text {mut }}=I D H$ mutant; $I D H^{\text {wt }}=I D H$ wild-type; WHO $=$ World Health Organization; HG $=2$-hydroxyglutarate; $\mathrm{nCET}=$ non-contrast-enhancing tumor; cIMPACT-NOW = Consortium to Inform Molecular and Practical Approaches to CNS Tumor Taxonomy

$\mathbf{R}$ adiogenomics, also known as imaging genomics, aims to predict genetic markers based on imaging features. An important change in the 2016 update to the World Health Organization (WHO) Classification of Tumors of the CNS was the addition of molecular markers to the diagnosis of diffuse gliomas, providing an integrated phenotypic and genetic classification. ${ }^{1}$ In particular, diffuse gliomas of all grades (II-IV) are now classified according to isocitrate dehydrogenase (IDH) status as either IDH mutant $\left(I D H^{\mathrm{mut}}\right)$ or $I D H$ wild-type $\left(I D H^{\mathrm{wt}}\right)$, and grade II and III gliomas with an $I D H$ mutation are further classified based on the presence or absence of $1 \mathrm{p} / 19 \mathrm{q}$-codeletion (with codeletion representing combined loss of both the short arm of chromosome 1 and the long arm of chromosome 19). ${ }^{2}$ The greater importance of genetic markers has led to a rapid growth in radiogenomics research in

Received April 29, 2020; accepted after revision June 27.

From the Departments of Cancer Imaging (A.L.) and Medical Oncology (M.A.R.), Peter MacCallum Cancer Centre, Melbourne, Australia; Sir Peter MacCallum Department of Oncology (A.L.), and Department of Surgery (K.J.D.), The University of Melbourne, Parkville, Australia; Departments of Anatomical Pathology (S.J.R.-T.), Neurology (A.N.), and Neurosurgery (K.J.D.), The Royal Melbourne Hospital, Parkville, Australia; and Department of Neuroscience, Faculty of Medicine (A.N.), Nursing and Health Sciences, Central Clinical School, Monash University, Melbourne, Australia.

Arian Lasocki was supported by a Peter MacCallum Cancer Foundation Discovery Partner Fellowship.

Please address correspondence to Arian Lasocki, Department of Cancer Imaging, Peter MacCallum Cancer Centre, Grattan St, Melbourne, Victoria 3000, Australia; e-mail: arian.lasocki@petermac.org

- Indicates open access to non-subscribers at www.ajnr.org

http://dx.doi.org/10.3174/ajnr.A6769 gliomas, with progressively increasing complexity, including the incorporation of artificial intelligence techniques.

Most radiogenomics research has focused on MR imaging, though a small minority of studies have considered CT (in particular in relation to the presence of calcification ${ }^{3,4}$ ), and there is also a growing interest in PET, in particular using amino acid tracers such as ${ }^{18}$ F-FDOPA (fluorine-18-fluoro-L-dihydroxyphenylalanine), ${ }^{5,6}$ MR imaging studies have examined both conventional MR imaging sequences (eg, pre- and postcontrast T1WI, T2WI, and FLAIR) and widely used advanced sequences (eg, DWI and MR imaging perfusion techniques). Notably, in addition to standard MR imaging spectroscopy ${ }^{7}$, novel spectroscopic assessment targeting 2-hydroxyglutarate $(2 \mathrm{HG})$ - an oncometabolite that accumulates in $\mathrm{IDH}^{\text {mut }}$ gliomas caused by catalysis of alpha-ketoglutarate to $2 \mathrm{HG}^{8}$ - has been shown to have high sensitivity for detecting IDH mutations. ${ }^{9,10}$ Newer MR imaging techniques such as chemical exchange-dependent saturation transfer imaging have also been examined ${ }^{11}$ but currently largely lie outside the clinical domain. A multiparametric approach improves accuracy, ${ }^{12,13}$ though a greater number of variables requires larger cohorts to provide a robust algorithm.

There has also been increasing interest in harnessing the power of a variety of computational techniques. Radiomics describes a range of computational methods to extract quantitative features from radiographic images ${ }^{11}$-most commonly MR imaging in the case of brain tumors-providing quantitative assessment of features that include signal intensity, shape, volume, and heterogeneity. ${ }^{14}$ These features, with the aid of machine learning methods, can in 
turn be mined to detect correlations with genomic markers. ${ }^{11}$ More recently, deep learning (a subset of machine learning ${ }^{15}$ ) using convolutional neural networks has further improved radiogenomic prediction. ${ }^{13,16}$ Initial concerns regarding the uninterpretable "black box" nature of neural networks are being overcome by more recent work; for example, Chang et $\mathrm{al}^{16}$ applied principal component analysis to the final feature vector to demonstrate the appearances most strongly correlating with a particular genomic marker.

Despite the rapid advances and growth in the complexity of the techniques being examined, there has been little translation into the clinical domain. This has resulted in a growing disconnect between cutting-edge research and assimilation into clinical practice, though the fundamental goal is for these techniques to improve patient care. Therefore, it is important to take a step back and discuss possible ways in which radiogenomics could be incorporated into clinical practice, with a multidisciplinary focus. By identifying relevant clinical scenarios, we also hope to encourage research into the clinical impact of radiogenomics, ideally prospectively. Being the key genetic markers in the current WHO classification, our discussion focuses on $I D H$ and $1 \mathrm{p} / 19 \mathrm{q}$ status, though similar principles generally apply also to other genetic markers. Our focus is on possible clinical scenarios rather than a detailed discussion on the specific imaging features.

\section{CLINICAL APPLICATIONS OF RADIOGENOMICS}

Definitive histopathologic and genetic assessment based on histologic specimens remains the criterion standard for the diagnosis of brain tumors, but imaging has several advantages. The most obvious limitation of histology is that it requires surgery-and its associated morbidity-to obtain the specimen, but imaging is noninvasive. Thus, radiogenomics has value before surgery or in situations when there may be a preference for or a possibility of avoiding surgery. A further advantage of imaging is its ability to image the entire tumor, which is of particular value for gliomas given their inherently diffuse and heterogeneous nature. Finally, radiogenomics can be tailored toward the clinical question, by the appropriate selection of imaging modalities, the optimal use of the given technique (eg, in the selection of MR imaging sequences or PET tracers), and even in the application of computational techniques. Possible clinical uses of radiogenomics are discussed, including the advantages and disadvantages of radiogenomics and a brief description of specific imaging features that could be used. The scenarios discussed include:

- Providing a "virtual biopsy."

- Aiding patient counseling.

- Guiding perioperative management.

- The prediction of "noncanonical" IDH mutations.

- Considerations in centers with limited access to genetic testing methods.

- Tumor reclassification.

- Overcoming the limitations of histologic assessment.

- Posttreatment follow-up.

\section{"Virtual Biopsy"}

The ultimate vision is, perhaps, for imaging genomics to deliver a "virtual biopsy," by providing-noninvasively—a sufficiently confident prediction of genetic status to guide clinical management.

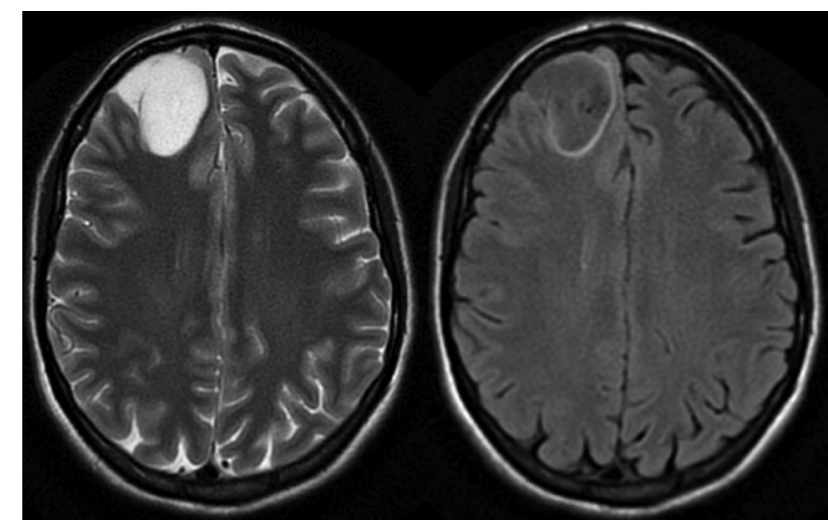

FIGURE. Axial T2WI (left) and FLAIR (right) image of a right frontal lobe WHO grade III, IDH-mutant, 1p/19q noncodeleted glioma demonstrating the T2-FLAIR mismatch sign.

Arguably, the T2-FLAIR mismatch sign, when present, already provides a confident prediction of an $I D H^{\text {mut }}, 1 \mathrm{p} / 19 \mathrm{q}$ noncodeleted tumor, having been shown to have a $100 \%$ positive predictive value in several different cohorts, with moderate to near-perfect interobserver agreement $(\kappa=0.56-0.88)^{4,12,17,18}$ (Figure). False-positive results can occur in the case of enhancing tumors or pediatric patients, ${ }^{19,20}$ but exceptions are rare in the case of nonenhancing tumors in an adult population, ${ }^{19}$ and this sign remains highly specific of an $I D H^{\text {mut }}, 1 \mathrm{p} / 19 \mathrm{q}$ noncodeleted tumor in this context. The main limitation of this sign, however, is its sensitivity, being present in $22 \%-51 \%$ of $I D H^{\text {mut }}, 1 \mathrm{p} / 19 \mathrm{q}$ noncodeleted lower-grade gliomas in different cohorts. $^{12,17,18}$

Even if imaging genomics were able to approach $100 \%$ accuracy more widely, however, surgery not only provides a diagnosis (both histologic and genetic) but is also a key therapeutic technique in diffuse gliomas of all grades. ${ }^{21,22}$ Indeed, the importance of surgery may increase because of the push toward extending resection beyond the enhancing component of gliomas to the nonenhancing tumor component. ${ }^{23-25}$ Furthermore, there has been an increasing push toward early functional-guided resection for lower-grade gliomas to optimize both oncologic and functional long-term outcomes. ${ }^{26}$ As such, other than for tumors in locations that are challenging surgically or in patients with a gliomatosis-like pattern in whom even subtotal resection is not feasible, ${ }^{26}$ resection cannot practically be avoided unless medical treatments improve dramatically.

Nevertheless, there are clinical scenarios when the differential diagnosis of a brain lesion includes non-neoplastic etiologies that do not require immediate surgery, in particular when the lesion is nonenhancing. A radiogenomic approach can aid decision making in such cases. The presence of imaging features that have been shown to correlate with a particular glioma genotype supports a diagnosis of a glioma, and thus a need for surgery, but the lack of such features gives clinicians greater confidence in managing the lesion as nongliomatous. Possible scenarios include focal lesional epilepsy caused by isolated amygdala enlargement with associated T2 hyperintensity ${ }^{27}$ and unilateral mesial temporal FLAIR hyperintensity that is suspected to be caused by autoimmune encephalitis. ${ }^{28}$ 


\section{Patient Counseling}

Radiogenomics is relevant for counseling patients before surgery. A "watch-and-wait" approach may have been used in the past for patients suspected on imaging of having a low-grade glioma but is going out of favor because of the importance of genetic markers and a recognition of the effect of extent of resection on survival. ${ }^{21}$ Patients may nevertheless wish to adopt such a strategy, for example, because of fear, logistic difficulties, or medical comorbidities, and the ability to predict the genetic status with MR imaging allows a more informed decision-making process. If MR imaging suggests an $I D H^{\mathrm{wt}}$ tumor, this provides further support for recommending surgery because tumor progression is likely to be more rapid, and it is important to confirm $I D H^{\mathrm{wt}}$ status to institute appropriate management. Even if the patient remains hesitant, imaging follow-up should occur at a shorter interval. In contrast, if an $I D H$ mutation can be confidently predicted based on the preoperative imaging (eg, because of the presence of a T2FLAIR mismatch sign ${ }^{4,17,18}$ or high ADC values), ${ }^{29}$ this may provide greater confidence that active surveillance is a feasible alternative. Such considerations already occur to some extent (eg, based on the presence or absence of enhancement), but the addition of radiogenomics data would further optimize personalized care.

\section{Perioperative Management}

Seizures are common in diffuse gliomas, particularly grade II-III tumors. ${ }^{30-32} \mathrm{IDH}$ mutations have been proposed as a potential biomarker of glioma-associated epilepsy, being associated with increased rates of seizures in both the pre- and postoperative settings. ${ }^{30,31,33} 2 \mathrm{HG}$ is structurally similar to the excitatory neurotransmitter glutamate and is hypothesized to activate excitatory N-Methyl-d-aspartate receptors. ${ }^{30}$ Radiogenomic identification of an $I D H^{\text {mut }}$ tumor thus suggests that patients could benefit from preoperative prophylactic antiepileptic treatment.

\section{Prediction of Noncanonical IDH Mutations}

After histology has confirmed a diffuse glioma, $I D H$ testing is the next step in the molecular characterization of both grade II or III gliomas and glioblastomas. ${ }^{2}$ Traditionally, $I D H$ testing has started with immunohistochemistry, and this remains the practice in most centers. An inherent limitation of this technique is that it is specific to the $\mathrm{R} 132 \mathrm{H}$ mutation in the IDH1 gene and is thus unable to detect "noncanonical" IDH mutations, namely, nonR132H-IDH1 mutations and all IDH2 mutations. ${ }^{34}$ The possibility of a noncanonical mutation is sufficiently high in grade II and III tumors ${ }^{34}$ that the WHO mandates definitive $I D H$ testing for these tumors. ${ }^{2}$ Definitive $I D H$ testing is not required for older patients ( 55 years of age and older) with a glioblastoma and negative IDH1 immunohistochemistry because of the very low likelihood of a noncanonical mutation. ${ }^{35}$ Chen et $\mathrm{al}^{36}$ have created and described an on-line tool that predicts, with high accuracy, the likelihood of a noncanonical $I D H$ mutation based on patient age; tumor grade; and, if a glioblastoma, whether there was a prior history of a grade II or III tumor (ie, whether the glioblastoma is "primary" or "secondary"). This allows a determination as to whether a noncanonical mutation is sufficiently likely to warrant definitive $I D H$ testing, and such a threshold can vary between individuals and departments. Radiogenomics has the potential to add to such a system, either based on simple criteria (eg, the likelihood of an IDH mutation is very low if a glioblastoma is located outside of the frontal lobes and demonstrates little non-contrast-enhancing tumor [nCET] $)^{37}$ or on more comprehensive radiomic assessment.

\section{Limited Access to Genetic Testing Methods}

Similar considerations are even more relevant if a center does not have ready access to all the testing methods required for complete characterization according to the WHO guidelines. IDH1 immunohistochemistry is relatively accessible, but testing for $1 \mathrm{p} / 19 \mathrm{q}$ status and, in particular, noncanonical $I D H$ mutations is not as widely available. Even if there is a possibility of sending specimens to another institution for testing, this adds to costs (to either the institution or the patient). Although the cost of performing any given test can be expected to decrease over time, there is likely to be a growth in the number of genetic markers incorporated into future diagnostic classifications. Arguably, this is less of an issue in glioblastoma because $I D H$ status currently provides important prognostic information but does not alter standard management (beyond the clinical trial setting). In the case of grade II and III gliomas, however, there is the potential for changes to management. For example, if chemotherapy is considered, whereas temozolomide is typically used for astrocytomas (whether $I D H^{\mathrm{mut}}$ or $I D H^{\mathrm{wt}}$ ), procarbazine, lomustine, and vincristine chemotherapy may be preferred for oligodendrogliomas, ${ }^{38}$ and $1 \mathrm{p} / 19 \mathrm{q}$ status is important for this distinction. Even if chemotherapy is not considered at the initial diagnosis, it is likely to be warranted at progression.

Radiogenomics has the potential to provide complementary information in such a setting and may more accurately predict the molecular subtype than the histologic phenotype if certain MR imaging features are present. ${ }^{4}$ It is rare for tumor operations to occur without preoperative MR imaging; therefore, radiogenomic information, at the very least involving a neuroradiologist's interpretation of conventional sequences, is likely to be available. This may be particularly useful for a smaller institution requesting advice from a quaternary center because the transfer of data is facilitated by the digital nature of modern imaging, especially compared with sending pathologic specimens for further analysis.

\section{Tumor Reclassification}

Many patients currently living with gliomas-in particular, with lower-grade gliomas-will have been diagnosed before the 2016 WHO update. Such patients may have an outdated pathologic diagnosis, which could change if the histology were to be reviewed. This is most common in tumors previously labeled oligoastrocytomas-a diagnosis that is now obsolete except in rare cases-with molecular markers allowing a definitive diagnosis as either an astrocytoma or oligodendroglioma. ${ }^{39}$ Also, an astrocytoma may occasionally be reclassified as an oligodendroglioma or vice versa. ${ }^{39}$ If tumor progression occurs and a change to the treatment is being considered, there is an opportunity to re-evaluate the diagnosis. Even if there is insufficient histologic material for retrospective testing, review of the patient's preoperative MR imaging may allow prediction of the molecular subtype without the need for repeat 
surgery. At the least, radiogenomics may suggest that the molecular subtype is different from what would be suspected from the histologic testing and thus provide a triaging role.

Such considerations can be expected to become more prevalent in the future as further genetic markers with prognostic and therapeutic implications are identified and potentially incorporated into subsequent classification systems. Indeed, the rapid growth-both recent and continuing-in our understanding of the molecular characteristics of CNS tumors has also lead to the development of cIMPACT-NOW (Consortium to Inform Molecular and Practical Approaches to CNS Tumor Taxonomy). ${ }^{40}$ Given that the 2016 WHO update occurred 9 years after the preceding classification, ${ }^{41}$ this initiative acknowledges a need to evaluate classification progress faster than can occur for a complete update to the WHO classification and thus provides consensus recommendations for proposed changes to future classifications. ${ }^{40}$ For example, cIMPACT-NOW has recommended that in the case of a phenotypic grade II or III astrocytoma with an IDH mutation, loss of nuclear ATRX expression, or diffuse p53 immunopositivity is adequate to label the tumor an $I D H^{\text {mut }}$ astrocytoma according to the current WHO guidelines without definitive $1 \mathrm{p} / 19 \mathrm{q}$ testing. ${ }^{42}$ Another important recommendation is the addition of the entity diffuse astrocytic glioma, $I D H^{\mathrm{wt}}$, with molecular features of glioblastoma. ${ }^{43}$ This suggests patients be treated as having glioblastoma even when the formal histologic criteria have not been met. ${ }^{43}$ This diagnosis requires an additional finding of high-level epidermal growth factor receptor (EGFR) amplification, whole-chromosome 7 gain and whole-chromosome 10 loss, or a TERT promoter mutation. ${ }^{43}$ Thus, markers beyond just $I D H$ and $1 \mathrm{p} / 19 \mathrm{q}$ status have become clinically relevant, providing new avenues for radiogenomics research and subsequent clinical integration before the next WHO update.

It is worthwhile noting that changes to classification systems also have implications for the relevance of previous research. For example, previously, grade III and IV gliomas were often grouped together as high-grade gliomas, with grade II gliomas considered low-grade gliomas. Now, grade II and III tumors are often considered together, sometimes referred to together as lower grade gliomas. As such, the value of previous studies comparing grade II and grade III/IV tumors is much diminished. If, for example, future iterations of the WHO classification were to put all emphasis on genotype, removing histologic grade entirely, current radiogenomic literature-which typically distinguishes between grade II/III and IV tumors, as per the current WHO classification-would need to be reassessed. An advantage of radiogenomics, however, is its ability to reanalyze previous data based on the most up-to-date information, and computational techniques are making such processes easier and faster.

\section{Overcoming Limitations of Histology}

Despite remaining the criterion standard for glioma diagnosis, histopathology has some limitations. $1 \mathrm{p} / 19 \mathrm{q}$ testing methods have been shown to occasionally provide false-positive results, for example, in the case of partial- rather than whole-arm deletion. ${ }^{44}$ Sampling error is a well-recognized issue in gliomas because of their heterogeneous nature, and this is particularly relevant to determining the grade ${ }^{45}$ but also potentially relevant to the determination of the genotype. Even advanced $I D H$ testing methods may produce false-negative results if there is a limited number of tumor cells within the sample being analyzed. ${ }^{34,46}$ In addition, although IDH status is generally considered to be consistent throughout a glioma, some heterogeneity can be observed. For example, Preusser et $\mathrm{al}^{46}$ reported that only a fraction of tumor cells exhibited immunostaining in approximately $15 \%$ of their glioma cohort, with some of these cases demonstrating biphasic immunostaining patterns. As such, there is the potential for MR imaging to provide genetic information when there is some reason to suspect inaccuracy of genetic testing. For example, if the pathologist acknowledges the possibility of a false-negative test result because of a small sample, imaging may be able to provide a confident prediction of the molecular status without the morbidity of repeat surgery. Equally, if a highly specific imaging feature is present but is at odds with the genetic testing result, in particular if genetic testing results are negative, more comprehensive histologic assessment may be warranted.

There are $2 \mathrm{IDH}$ alleles, and mutation in 1 is sufficient for a tumor to be considered $I D H^{\text {mut } 34}$ Occasions exist, however, when tumors express only 1 of the 2 alleles, known as monoallelic gene expression. ${ }^{34}$ This can occur in $I D H^{\text {mut }}$ gliomas, with an incidence of $15 \%$ in 1 study. ${ }^{47}$ Most commonly, only the $I D H^{\mathrm{wt}}$ allele is expressed, resulting in a worse prognosis. ${ }^{47}$ Similarly, $I D H^{\text {mut }}$ gliomas may rarely lose their $I D H^{\text {mut }}$ allele. ${ }^{48}$ As such, there is the potential for $I D H^{\text {mut }}$ gliomas to be or become functionally $I D H^{\mathrm{wt}}$, and this may not be readily identifiable by genetic testing methods. ${ }^{34}$ Radiogenomics could have value in such situations, potentially identifying an MR imaging appearance more in keeping with an $I D H^{\mathrm{wt}}$ rather than $I D H^{\mathrm{mut}}$ tumor, and thus suggesting a more aggressive treatment approach. Interestingly, deletion of the wild-type allele in $I D H^{\text {mut }}$ gliomas also results in decreased $2 \mathrm{HG}$ production, showing that both wild-type and mutant alleles are necessary for $2 \mathrm{HG}$ production in glioma cells. ${ }^{49}$ Thus, although $2 \mathrm{HG}$ spectroscopy has typically been studied in the preoperative setting, ${ }^{9,50}$ it may also be able to quantitatively demonstrate such changes in $I D H$ expression and $2 \mathrm{HG}$ production as the tumor evolves.

Similar considerations are also relevant to mixed tumors. Although an important role of the new WHO classification is to largely remove the diagnostic uncertainly of tumors with a mixed histologic phenotype (in particular oligoastrocytomas), true genetically mixed tumors-with 2 genetically distinct components-do occasionally occur. ${ }^{51,52}$ Genetic testing is typically performed in a single portion of the tumor, so the incidence of true mixed tumors may be underappreciated. ${ }^{51}$ Radiogenomics therefore has the potential to identify distinct appearances in different portions of the tumor, suggesting value in repeating testing in a different portion.

It has become critical for medical oncologists and neurosurgeons treating patients with gliomas to have a strong understanding of the molecular subtypes of gliomas and the histologic testing methods. This knowledge is also becoming increasingly important for neuroradiologists, and it may be worthwhile not only stating the likely diagnosis in the radiologic report but also the likely molecular subtype if there are indicative features. Similarly, there is value in neuropathologists having some knowledge of the growing field of radiogenomics, at least the imaging 
features most sensitive or specific for a particular molecular subtype. This may allow neuropathologists to appropriately temper their confidence if relevant radiologic features are present and there is some doubt as to the validity of the histologic assessment, facilitating optimal multidisciplinary care.

\section{Posttreatment Follow-Up}

As discussed, resection is a key part of the patient's management at diagnosis, which somewhat decreases the value of radiogenomics at this time. Posttreatment follow-up could therefore be a more useful clinical context for radiogenomics because further surgery may not be otherwise warranted. This is particularly relevant given that the newly understood genetic landscape of gliomas has progressed to the therapeutic domain, for example, with the advent of $I D H$ inhibitors, which have now entered clinical trials. ${ }^{53}$ Intuitively, knowledge of the imaging appearance associated with the genetic mutation being targeted therapeutically has value. For example, features associated with an $I D H$ mutation in glioblastoma include the presence of nCET, ${ }^{54}$ a masslike morphology of $\mathrm{nCET}^{55}$ and a minimally invasive phenotype on diffusion-tensor imaging. ${ }^{56}$ Targeted therapies have produced dramatic responses in some extracranial malignancies, but the plasticity of tumor cells can result in resistance to these treatments through a variety of mechanisms, ${ }^{57}$ and similar mechanisms can be expected in gliomas. The imaging changes related to targeted treatments such as $I D H$ inhibitors are as yet unknown, but given that gliomas have strategies to circumvent $I D H$ mutation as outlined earlier, one would expect that these strategies would occur more commonly on treatment with a targeted agent. Again, if the appearance of the tumor were to change, for example, by developing a more infiltrative imaging phenotype, this could suggest the development of resistance to the therapeutic agent.

Of note, much of the research in this field focuses on preoperative imaging and cannot necessarily be extrapolated to the posttreatment setting. A further difficulty with the posttreatment context is that a variety of additional confounders are introduced. There are differences in the extent of resection and postsurgical changes, and imaging changes after radiation therapy can also vary between patients. These complexities are most pronounced with medical therapies, including conventional chemotherapy (in particular temozolomide), antiangiogenics agents (the most commonly used being bevacizumab), and less established treatments such as immunotherapy, vaccine therapy, and, increasingly, targeted agents. These treatments have revealed challenging imaging patterns, well demonstrated by the phenomena of pseudoprogression and pseudoresponse, and one would expect that emerging and future therapies may provide similar conundrums. With the greater number of potential variables in the posttreatment setting, development of a robust radiogenomic algorithm requires a much larger number of testing cases, providing an opportunity for automated computational techniques. Advanced artificial intelligence techniques such as deep learning are also able to identify features not readily discernible to the human eye, of particular relevance given that important clinical scenarios such as pseudoprogression are notoriously difficult for neuroradiologists.

\section{CHOOSING APPROPRIATE RADIOGENOMIC FEATURES}

The way in which radiogenomic data are incorporated into clinical practice may vary depending on the specific scenario. For example, if the hope is to confidently diagnose a specific mutation or molecular subtype, specificity is important. The T2-FLAIR mismatch sign is the best example of such a feature, being able to predict a $I D H^{\text {mut }}, 1 \mathrm{p} / 19 \mathrm{q}$ noncodeleted grade II/III tumor with high confidence. ${ }^{4,12,17,18}$ If this feature is absent, however, its sensitivity is insufficient to exclude an $I D H^{\text {mut }}, 1 \mathrm{p} / 19 \mathrm{q}$ noncodeleted tumor. ${ }^{4,17,18}$ In contrast, although the presence of nCET is associated with $I D H$ mutations in glioblastoma, ${ }^{54}$ it is not sufficiently specific to confidently predict an $I D H$ mutation; ${ }^{37}$ rather, the moderate sensitivity of this feature means that a glioblastoma without substantial nCET is unlikely to have an IDH mutation (ie, it is likely $\left.I D H^{\mathrm{wt}}\right) .{ }^{37}$ Thus, to confidently predict a particular genetic marker, specificity is important, but if the goal is to exclude a genetic marker, sensitivity is more important.

The specific clinical scenario has implications for the use of the results of radiogenomics research. Because there is usually a degree of overlap in the imaging features between molecular subtypes, many studies aim to maximize the overall accuracy by using area under the curve analysis, but this may not be optimal for a given patient. If such a model were to predict a given genetic marker in a specific patient, it would be important to know not just the likely result but also the degree of confidence in this result because this would greatly impact the value of this information for any given patient. Some continuous variables, such as ADC, can potentially be thresholded in different ways depending on how they are to be used. $I D H^{\mathrm{wt}}$ gliomas are associated with lower ADC values, but there is overlap with $I D H^{\text {mut }}$ gliomas, in particular at intermediate ADC values. ${ }^{29,58,59}$ An appropriate threshold can be selected depending on whether there is a desire to more confidently diagnose or exclude a particular mutation. For example, if the goal were to confidently diagnose an $I D H$ mutation, a higher ADC threshold would be preferable, with ADC values above this being particularly suggestive of an $I D H^{\text {mut }}$ tumor. Multivariate methods such as radiomics have even greater potential for being targeted toward optimizing sensitivity or specificity depending on the clinical scenario.

Here, it is important to recognize that whether a feature can be used and how this could be done depends on the grade of the tumor. For example, the T2-FLAIR mismatch sign and the presence of calcifications (predictive of an $I D H^{\text {mut }}, 1 \mathrm{p} / 19 \mathrm{q}$ codeleted tumor) are only relevant to grade II and III tumors. ${ }^{3,4,12,17,18}$ In contrast, a frontal lobe location is associated with $I D H$ mutations in both grade II and III gliomas and glioblastomas, ${ }^{37,54,60}$ but the positive predictive value varies between grades because of the much lower incidence of $I D H$ mutations in glioblastoma compared with grade II and III gliomas. ${ }^{34}$

\section{CONCLUSIONS}

The field of radiogenomics has advanced rapidly in recent times because of the increased understanding of the importance of molecular markers and the development of computational techniques. The clinical implementation of these techniques is lagging behind the research, however, and this disconnect warrants further 
consideration. Possible roles for radiogenomics include determining the likely molecular subtype in gliomas when complete genetic characterization is not practical, reclassifying tumors diagnosed before the current classification systems, and optimizing discussion with patients when conservative management is being considered. With the advent of targeted therapies for glioma, there is also the potential to identify features suggestive of resistance to treatment.

Disclosures: Arian Lasocki—RELATED: Grant: Peter MacCallum Cancer Foundation, Comments: Supported by a Peter MacCallum Cancer Foundation Discovery Partner Fellowship.* *Money paid to the institution.

\section{REFERENCES}

1. Louis DN, Perry A, Reifenberger G, et al. The 2016 World Health Organization Classification of Tumors of the Central Nervous System: a summary. Acta Neuropathol 2016;131:803-20 CrossRef Medline

2. Louis DO, Wiestler OD, Cavanee WK. World Health Organization Histological Classification of Tumours of the Central Nervous System. International Agency for Research on Cancer; 2016

3. Saito T, Muragaki Y, Maruyama T, et al. Calcification on CT is a simple and valuable preoperative indicator of $1 \mathrm{p} / 19 \mathrm{q}$ loss of heterozygosity in supratentorial brain tumors that are suspected grade II and III gliomas. Brain Tumor Pathol 2016;33:175-82 CrossRef Medline

4. Lasocki A, Gaillard F, Gorelik A, et al. MRI features can predict 1p/19q status in intracranial gliomas. AJNR Am J Neuroradiol 2018;39:687-92 CrossRef Medline

5. Cicone F, Carideo L, Scaringi C, et al. (18)F-DOPA uptake does not correlate with IDH mutation status and $1 \mathrm{p} / 19 \mathrm{q}$ co-deletion in glioma. Ann Nucl Med 2019;33:295-302 CrossRef Medline

6. Verger A, Metellus $\mathrm{P}$, Sala Q, et al. IDH mutation is paradoxically associated with higher (18)F-FDOPA PET uptake in diffuse grade II and grade III gliomas. Eur J Nucl Med Mol Imaging 2017;44:130611 CrossRef Medline

7. Nakae S, Murayama K, Sasaki H, et al. Prediction of genetic subgroups in adult supra tentorial gliomas by pre- and intraoperative parameters. J Neurooncol 2017;131:403-12 CrossRef Medline

8. Dang L, White DW, Gross S, et al. Cancer-associated IDH1 mutations produce 2-hydroxyglutarate. Nature 2009;462:739-44 CrossRef Medline

9. Choi C, Ganji SK, DeBerardinis RJ, et al. 2-hydroxyglutarate detection by magnetic resonance spectroscopy in IDH-mutated patients with gliomas. Nat Med 2012;18:624-29 CrossRef Medline

10. Suh $\mathrm{CH}$, Kim HS, Jung SC, et al. Imaging prediction of isocitrate dehydrogenase (IDH) mutation in patients with glioma: a systemic review and meta-analysis. Eur Radiol 2019;29:745-58 CrossRef Medline

11. Jiang S, Zou T, Eberhart CG, et al. Predicting IDH mutation status in grade II gliomas using amide proton transfer-weighted (APTw) MRI. Magn Reson Med 2017;78:1100-09 CrossRef Medline

12. Batchala PP, Muttikkal TJE, Donahue JH, et al. Neuroimaging-based classification algorithm for predicting $1 \mathrm{p} / 19 \mathrm{q}$-codeletion status in IDH-mutant lower grade gliomas. AJNR Am J Neuroradiol 2019;40:42632 CrossRef Medline

13. Li Z, Wang Y, Yu J, et al. Deep learning based radiomics (DLR) and its usage in noninvasive IDH1 prediction for low grade glioma. Sci Rep 2017;7:5467 CrossRef Medline

14. Gillies RJ, Kinahan PE, Hricak H. Radiomics: images are more than pictures, they are data. Radiology 2016;278:563-77 CrossRef Medline

15. Rudie JD, Rauschecker AM, Bryan RN, et al. Emerging applications of artificial intelligence in neuro-oncology. Radiology 2019;290:60718 CrossRef Medline

16. Chang P, Grinband J, Weinberg BD, et al. Deep-learning convolutional neural networks accurately classify genetic mutations in gliomas. AJNR Am J Neuroradiol 2018;39:1201-07 CrossRef Medline
17. Patel SH, Poisson LM, Brat DJ, et al. T2-FLAIR mismatch, an imaging biomarker for IDH and $1 \mathrm{p} / 19 \mathrm{q}$ status in lower-grade gliomas: a TCGA/TCIA project. Clin Cancer Res 2017;23:6078-85 CrossRef Medline

18. Broen MPG, Smits M, Wijnenga MMJ, et al. The T2-FLAIR mismatch sign as an imaging marker for non-enhancing IDH-mutant, 1p/19q-intact lower-grade glioma: a validation study. Neuro-Oncol 2018;20:1393-99 CrossRef Medline

19. Johnson DR, Kaufmann TJ, Patel SH, et al. There is an exception to every rule-T2-FLAIR mismatch sign in gliomas. Neuroradiology 2019;61:225-27 CrossRef Medline

20. Juratli TA, Tummala SS, Riedl A, et al. Radiographic assessment of contrast enhancement and T2/FLAIR mismatch sign in lower grade gliomas: correlation with molecular groups. J Neurooncol 2019;141:327-35 CrossRef Medline

21. Schiff D, van den Bent M, Vogelbaum MA, et al. Recent developments and future directions in adult lower-grade gliomas: Society for Neuro-Oncology (SNO) and European Association of Neuro-Oncology (EANO) consensus. Neuro Oncol 2019;21:837-53 CrossRef

22. Weller M, van den Bent M, Hopkins K, European Association for Neuro-Oncology (EANO) Task Force on Malignant Glioma, et al. EANO guideline for the diagnosis and treatment of anaplastic gliomas and glioblastoma. Lancet Oncol 2014;15:e395-403 CrossRef Medline

23. Li YM, Suki D, Hess $\mathrm{K}$, et al. The influence of maximum safe resection of glioblastoma on survival in $\mathbf{1 2 2 9}$ patients: can we do better than gross-total resection? J Neurosurg 2016;124:977-88 CrossRef Medline

24. Pessina F, Navarria P, Cozzi L, et al. Maximize surgical resection beyond contrast-enhancing boundaries in newly diagnosed glioblastoma multiforme: is it useful and safe? A single institution retrospective experience. J Neurooncol 2017;135:129-39 CrossRef Medline

25. Lasocki A, Gaillard F. Non-contrast-enhancing tumor: a new frontier in glioblastoma research. AJNR Am J Neuroradiol 2019;40:75865 CrossRef Medline

26. Duffau H. Diffuse low-grade glioma, oncological outcome and quality of life: a surgical perspective. Curr Opin Oncol 2018;30:38389 CrossRef Medline

27. Beh SMJ, Cook MJ, D'Souza WJ. Isolated amygdala enlargement in temporal lobe epilepsy: a systematic review. Epilepsy Behav 2016;60:3341 CrossRef Medline

28. Graus F, Titulaer MJ, Balu R, et al. A clinical approach to diagnosis of autoimmune encephalitis. Lancet Neurol 2016;15:391-404 CrossRef Medline

29. Thust SC, Hassanein S, Bisdas S, et al. Apparent diffusion coefficient for molecular subtyping of non-gadolinium-enhancing WHO grade II/III glioma: volumetric segmentation versus twodimensional region of interest analysis. Eur Radiol 2018;28:377988 CrossRef Medline

30. Chen H, Judkins J, Thomas C, et al. Mutant IDH1 and seizures in patients with glioma. Neurology 2017;88:1805-13 CrossRef Medline

31. Neal A, Kwan P, O'Brien TJ, et al. IDH1 and IDH2 mutations in postoperative diffuse glioma-associated epilepsy. Epilepsy Behav 2018;78:30-36 CrossRef Medline

32. Neal A, Morokoff A, O'Brien TJ, et al. Postoperative seizure control in patients with tumor-associated epilepsy. Epilepsia 2016;57:177988 CrossRef Medline

33. Liubinas SV, D'Abaco GM, Moffat BM, et al. IDH1 mutation is associated with seizures and protoplasmic subtype in patients with low-grade gliomas. Epilepsia 2014;55:1438-43 CrossRef Medline

34. Horbinski C. What do we know about IDH1/2 mutations so far, and how do we use it? Acta Neuropathol 2013;125:621-36 CrossRef Medline

35. Louis DN, Wesseling $P$, Paulus W, et al. cIMPACT-NOW update 1: not otherwise specified (NOS) and not elsewhere classified (NEC). Acta Neuropathol 2018;135:481-84 CrossRef Medline 
36. Chen L, Voronovich Z, Clark K, et al. Predicting the likelihood of an isocitrate dehydrogenase $\mathbf{1}$ or $\mathbf{2}$ mutation in diagnoses of infiltrative glioma. Neuro Oncol 2014;16:1478-83 CrossRef Medline

37. Lasocki A, Tsui A, Gaillard F, et al. Reliability of noncontrastenhancing tumor as a biomarker of IDH1 mutation status in glioblastoma. J Clin Neurosci 2017;39:170-75 CrossRef Medline

38. Weller M, van den Bent M, Tonn JC, et al. European Association for Neuro-Oncology (EANO) guideline on the diagnosis and treatment of adult astrocytic and oligodendroglial gliomas. Lancet Oncol 2017;18:e315-29 CrossRef Medline

39. Rogers TW, Toor G, Drummond K, et al. The 2016 revision of the WHO Classification of Central Nervous System Tumours: retrospective application to a cohort of diffuse gliomas. J Neurooncol 2018;137:181-89 CrossRef Medline

40. Louis DN, Aldape K, Brat DJ, et al. Announcing cIMPACT-NOW: the Consortium to Inform Molecular and Practical Approaches to CNS Tumor Taxonomy. Acta Neuropathol 2017;133:1-3 CrossRef Medline

41. Louis DN, Wiestler OD, Cavanee WK. World Health Organization histological classification of tumours of the central nervous system. International Agency for Research on Cancer, Lyon; 2007

42. Louis DN, Giannini C, Capper D, et al. cIMPACT-NOW update 2: diagnostic clarifications for diffuse midline glioma, H3 K27M-mutant and diffuse astrocytoma/anaplastic astrocytoma, IDH-mutant. Acta Neuropathol 2018;135:639-42 CrossRef Medline

43. Brat DJ, Aldape K, Colman H, et al. cIMPACT-NOW update 3: recommended diagnostic criteria for "Diffuse astrocytic glioma, IDH-wildtype, with molecular features of glioblastoma, WHO grade IV." Acta Neuropathol 2018;136:805-10 CrossRef Medline

44. Horbinski C, Nikiforova MN, Hobbs J, et al. The importance of $\mathbf{1 0 q}$ status in an outcomes-based comparison between $1 \mathrm{p} / 19 \mathrm{q}$ fluorescence in situ hybridization and polymerase chain reaction-based microsatellite loss of heterozygosity analysis of oligodendrogliomas. J Neuropathol Exp Neurol 2012;71:73-82 CrossRef Medline

45. Lasocki A, Tsui A, Tacey MA, et al. MRI grading versus histology: predicting survival of World Health Organization grade II-IV astrocytomas. AJNR Am J Neuroradiol 2015;36:77-83 CrossRef Medline

46. Preusser M, Wohrer A, Stary S, et al. Value and limitations of immunohistochemistry and gene sequencing for detection of the IDH1-R132H mutation in diffuse glioma biopsy specimens. $J$ Neuropathol Exp Neurol 2011;70:715-23 CrossRef Medline

47. Walker EJ, Zhang C, Castelo-Branco P, et al. Monoallelic expression determines oncogenic progression and outcome in benign and malignant brain tumors. Cancer Res 2012;72:636-44 CrossRef Medline
48. Pusch S, Sahm F, Meyer J, et al. Glioma IDH1 mutation patterns off the beaten track. Neuropathol Appl Neurobiol 2011;37:428-30 CrossRef Medline

49. Jin G, Reitman ZJ, Duncan CG, et al. Disruption of wild-type IDH1 suppresses D-2-hydroxyglutarate production in IDH1-mutated gliomas. Cancer Res 2013;73:496-501 CrossRef Medline

50. Tietze A, Choi C, Mickey B, et al. Noninvasive assessment of isocitrate dehydrogenase mutation status in cerebral gliomas by magnetic resonance spectroscopy in a clinical setting. J Neurosurg 2018;128:391-98 CrossRef Medline

51. Huse JT, Diamond EL, Wang L, et al. Mixed glioma with molecular features of composite oligodendroglioma and astrocytoma: a true “oligoastrocytoma"? Acta Neuropathol 2015;129:151-53 CrossRef Medline

52. Wilcox P, Li CC, Lee M, et al. Oligoastrocytomas: throwing the baby out with the bathwater? Acta Neuropathol 2015;129:147-49 CrossRef Medline

53. Huang $\mathrm{J}, \mathrm{Yu} \mathrm{J}, \mathrm{Tu} \mathrm{L}$, et al. Isocitrate dehydrogenase mutations in glioma: from basic discovery to therapeutics development. Front Oncol 2019;9:506 CrossRef Medline

54. Carrillo JA, Lai A, Nghiemphu PL, et al. Relationship between tumor enhancement, edema, IDH1 mutational status, MGMT promoter methylation, and survival in glioblastoma. AJNR Am J Neuroradiol 2012;33:1349-55 CrossRef Medline

55. Lasocki A, Gaillard F, Tacey M, et al. Morphologic patterns of noncontrast-enhancing tumor in glioblastoma correlate with IDH1 mutation status and patient survival. J Clin Neurosci 2018;47:16873 CrossRef Medline

56. Price SJ, Allinson $\mathrm{K}$, Liu $\mathrm{H}$, et al. Less invasive phenotype found in isocitrate dehydrogenase-mutated glioblastomas than in isocitrate dehydrogenase wild-type glioblastomas: a diffusion-tensor imaging study. Radiology 2017;283:215-21 CrossRef Medline

57. Ramos P, Bentires-Alj M. Mechanism-based cancer therapy: resistance to therapy, therapy for resistance. Oncogene 2015;34:3617-26 CrossRef Medline

58. Leu K, Ott GA, Lai A, et al. Perfusion and diffusion MRI signatures in histologic and genetic subtypes of WHO grade II-III diffuse gliomas. J Neurooncol 2017;134:177-88 CrossRef Medline

59. Xing Z, Yang X, She D, et al. Noninvasive assessment of IDH mutational status in World Health Organization grade II and III astrocytomas using DWI and DSC-PWI combined with conventional MR imaging. AJNR Am J Neuroradiol 2017;38:1138-44 CrossRef Medline

60. Darlix A, Deverdun J, Menjot de Champfleur N, et al. IDH mutation and $1 \mathrm{p} 19 \mathrm{q}$ codeletion distinguish two radiological patterns of diffuse low-grade gliomas. J Neurooncol 2017;133:37-45 CrossRef Medline 